
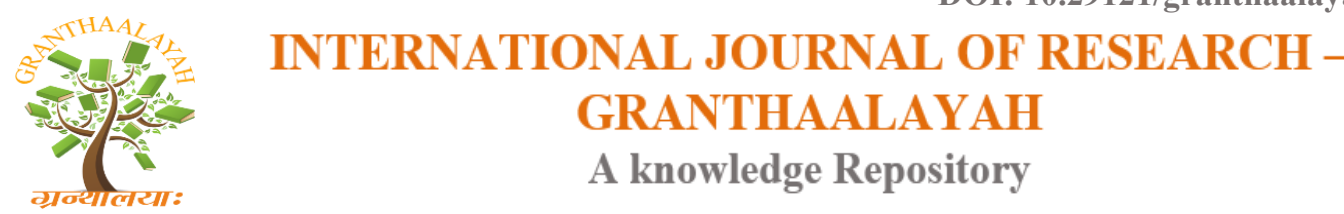

Science

\title{
A METHODOLOGY FOR REDUCING ENERGY UTILIZATION IN DENSE WIRELESS SENSOR NETWORKS
}

\author{
Senthil Kumar T ${ }^{1}$, Boselin Prabhu S $\mathbf{R}^{2}$, Rajkumar $\mathbf{R}^{3}$, Dr. S. Sophia ${ }^{4}$ \\ 1,2,3 Assistant Professor, SVS College of Engineering, Coimbatore, INDIA \\ ${ }^{4}$ Assistant Professor, Department of Electronics and Communication Engineering, Sri Krishna \\ College of Engineering and Technology, Coimbatore, INDIA
}

\begin{abstract}
The development of wireless sensor networks was motivated by military applications such as battlefield surveillance; today such networks are used in many industrial and consumer applications, such as industrial process monitoring and control, machine health monitoring, and so on. Wireless sensor Networks (WSNs), is one of the most rapidly growing scientific domain. This is because of the development of advanced sensor nodes with extremely low cost and the potential application of such sensor nodes are ever growing. One of the characteristic feature of WSNs compared to the traditional wireless communication networks, is the power awareness, due to the fact that the batteries of the sensor nodes have restricted lifetime and are difficult to be replaced. This is why we focus on power awareness, while the traditional wireless networks mainly focuses on the QOS. A typical sensor node consumes most of its energy during communication. However, energy expenditure takes place while performing sensing and data processing too. This work suggests the development of an advanced hierarchical routing technique, which gives improved performance over the existing techniques. Power consumption is highly reduced, thereby greatly reducing the cost of network and hence the lifetime of nodes can be greatly improved.
\end{abstract}

Keywords:

Hierarchical routing, cluster head, residual energy and threshold.

Cite This Article: Senthil Kumar T, Boselin Prabhu S R, Rajkumar R, and Dr. S. Sophia, "A METHODOLOGY FOR REDUCING ENERGY UTILIZATION IN DENSE WIRELESS SENSOR NETWORKS" International Journal of Research - Granthaalayah, Vol. 4, No. 1 (2016): 125-130.

\section{INTRODUCTION}

A Wireless Sensor Network [1] consists of a group of spatially distributed sensor nodes which are interconnected without using wires. Each of the distributed senor nodes typically consists of one or more sensing elements, a data processing unit, communicating components and a power source, which is usually a battery. The sensed data is collected, processed and then routed to the 
desired end user through a designated sink point, referred as base station. Now it has become feasible to construct multifunctional sensor nodes with advanced capabilities. Such sensor nodes are relatively of smaller size, lower cost and lesser power consumption. WSNs are originally motivated for the use in military applications, such as border monitoring. Now a day it is mainly focused on civilian applications such as environment monitoring, object tracking and biomedical applications.

\section{RELATED WORKS}

WSNs consist of hundreds of even thousands of sensor nodes which may be sparsely distributed in remote locations. Thus, it becomes infeasible to recharge or replace the dead batteries of the nodes. As soon as, some of the sensor nodes in a WSN [2] run out of energy, they stop functioning causing progressive deconstruction of the network. Therefore, one of the most stringent limitations that the development of a WSN faces is that of power consumption. Hence each and every protocol should be so designed, that minimum energy should be consumed during sensing, processing and communication. Three layers are involved in the functioning of a WSN. Physical and data link layers, of the protocol stack deals with energy awareness, radio communication hardware, low duty cycle issues, system partitioning and energy aware MAC protocols. At the Network layer, of the protocol stack, the main aim is to find ways for energy efficient route setup and reliable data transmission from the sensor nodes to the base station in order to prolong the overall network lifetime as much as possible . The routing protocols proposed have is the Hierarchical routing protocols. The main idea is that, every sensor node with in a WSN is grouped along with some other of its neighboring nodes so as to constitute a specific cluster. Data collected by the sensor nodes are not directly transmitted to the Base station. Instead, a node of the cluster called Cluster head, collects these data and forwards them to the base station after possibly having performed appropriate data aggregation. The major hierarchical routing algorithms [3] for sensor networks are LEACH, TEEN and SHPER.

The initial step in the generation of LEACH (Low Energy Adaptive clustering of Hierarchy), is the formation of clusters. More precisely, each sensor nodes decides whether or not to become the cluster head for the current round. The decision is based on the priority and also on the number to time the node has been a cluster head so for. The cluster nodes collect the data and send them to the cluster head. The radio to each cluster nodes can be turned off when there is no sensing takes place. When all the data have been received the cluster head aggregates the data in to a single composite signal. The composite signal is sent to the base station. The SHPER (Scaling Hierarchical Power efficient Routing) protocol includes base station and sensor nodes which are randomly distributed over a bounded area of interest. Both the Base station and sensor nodes are found to be stationary. The end users can access the data from the base station, which is located far away from the sensor field. All the cluster nodes are grouped in to cluster. Within each cluster, one of the cluster nodes is elected to be the cluster head. The election of cluster heads in SHPER is based purely on the residual energy.

In case of TEEN (Threshold sensitive Energy Efficient Network) protocol, the initial stage is the formation of clusters. In this mechanism each cluster member nodes becomes cluster head for a time interval called cluster period. The cluster heads that are nearer to the base station, that can communicate with the base station with reasonable power consumption is considered to the 
highest level cluster head. Similarly, the cluster head which is located far away from the base station is considered to be the lowest level cluster head. The operation of SHPER, protocol includes two main phases namely the initialization phase and the steady state phase. The Base station decides which node should be a cluster head. The nodes other than cluster head becomes cluster nodes. Each cluster head, along with some cluster nodes are grouped together to form a specific cluster.

The Base station sends the ID of each cluster heads which are newly elected. After that each node decides, to which cluster it belongs to and it informs its cluster head that, it will be a member of that cluster. The cluster head informs each of its cluster nodes when it can transmit. Accordingly the data is collected by the cluster head and aggregated, further being transmitted to the base station

\section{LIMITATIONS OF EXISTING SYSTEM}

LEACH [4] protocol has the disadvantage, when periodic transmissions are unnecessary, thus causing pointless power consumption. The election of cluster head is based on priority, and hence there is a possibility for weaker nodes to be drained because they are elected to be cluster heads as frequently as the stronger nodes. Moreover the protocol is based on the assumptions that all nodes begin with the same amount of energy capacity in each election round and all the nodes can transmit with enough power to reach the base station if needed. Nevertheless, in many cases these assumptions are unrealistic. TEEN [5] protocol has been developed for reactive networks so as to respond to sudden changes in the sensed attributes. This makes it appropriate for time critical applications. However, TEEN [11] is not suitable for applications where periodic reports are needed. In case of SHPER [6], the election of cluster heads is purely based on the base station. Hence unnecessary transmissions occur between the base station and cluster heads. Also the base station should keep track on the sensor nodes in order to decide which node has the highest residual energy. Hence unnecessary transmissions occur between the base station and cluster nodes, thereby causing increased power consumption.

\section{FEATURES OF PROPOSED SYSTEM}

This work suggests a new idea over the existing techniques. In case of existing techniques, the election of cluster heads and cluster nodes are entirely done by the base station. Hence they require additional power consumption. The work mainly suggests the cluster head to be completely responsible for all the process including the election of cluster heads and cluster nodes. The cluster head normally calculates the power consumed by the nodes which normally depends on the available power at the nodes and distance between the nodes and the cluster head. Two thresholds are used namely hard threshold and soft threshold. Hard threshold is the minimum possible value, of an attribute to trigger a sensor node to switch on its transmitter and transmit to the cluster head. Soft threshold is a small change in the value of the sensed attribute that triggers the node to switch on its transmitter and transmit data. The hard threshold [7] tries to reduce the number of transmission by allowing their nodes to transmit only when the sensed attribute is beyond a critical value .In a similar way, the soft threshold further reduces the number of transmissions that might have otherwise occurred when there is little or no change in the sensed attribute. At every cluster change the values of both the thresholds can be changed 
and thus enabling the user to control the tradeoff between energy efficiency and data accuracy. The cluster nodes [8] transmit the sensed data to the cluster head.

The main characteristic feature of this method is that, residual energy is transmitted along with the sensed data by the cluster nodes to the cluster head. The cluster head only transmits the aggregated data to the base station. Moreover every process such as initialization, formation of clusters, election of cluster heads and monitoring the residual energy [9] are entirely done by the cluster head. Store and forward technique is followed at the cluster head, so that the sensed attribute along with the residual energy [10] is collected from the cluster nodes, stored at the cluster head, further the aggregated data alone is forwarded to the base station. Since the base station has no direct link with the cluster nodes, unnecessary transmissions [16] are avoided, thereby saving enormous power. The simulation is performed by using the GloMoSim Simulator.

\section{BASIC CONCEPT OF THE SCHEME}

As described, cluster heads need to be evenly distributed over the whole network for saving energy [17]. In our scheme, we try to avoid redundant creation of cluster heads in a small area. The main activities in the set-up phase are election of candidate nodes, selection of cluster heads, scheduling at each cluster, and discovery of cluster head for $\mathrm{CH}$-to- $\mathrm{CH}$ data transmission. During set-up phase, each node first decides whether or not it can become a candidate node in each region for the current round. This decision is based on the value of the threshold T(n) as used in LEACH protocol. As seen in Equation1, $\mathrm{p}$ should be given a large value in order to elect many candidate nodes. Cluster heads are elected among the candidate nodes. We utilize an advertisement message to elect cluster heads. For this, the candidate nodes use a CSMA MAC protocol. Each candidate node broadcasts an advertisement message within its transmission range. This range is dependent on the maximum distance between the levels. In our scheme, the advertisement range is given double of the maximum distance (about $55 \mathrm{~m}$ ) to cover other levels. When a candidate node is located within $\mathrm{a} \times$ Advertisement Range where the value of a is predetermined between 0 and 1, it has to give up qualification of candidate node and has to stop joining the competition. Ordinary node, by contrast, decides the cluster to which it will belong for this round. This decision is based on the signal strength of the advertisement message. After each node has decided to which cluster it belongs, node must transmit its data to the appropriate cluster head (which is a member of the same cluster). After cluster head receives all the messages from the nodes that would like to be included in the cluster and based on the number of nodes contained in the cluster, the cluster head creates a TDMA schedule and assigns each node a time slot when it can transmit.

Each cluster head broadcasts this schedule back to the nodes in the cluster. Finally, cluster heads form $\mathrm{CH}$-to- $\mathrm{CH}$ routing path for data transmission of the steady-state phase. After schedule creation, each cluster head performs cluster head discovery to find an upward cluster head to reach the sink. For this, each cluster head utilizes two-way handshake technique, with REQ and ACK message. Each cluster head broadcasts REQ message within advertisement range. Upward cluster head receiving this REQ message transmits ACK message back to the cluster head that transmitted REQ message. When the node that transmitted REQ message receives ACK message, it chooses this cluster head which transmitted ACK message as the next hop. If cluster head cannot find upward cluster head, it chooses the sink as the next hop. 


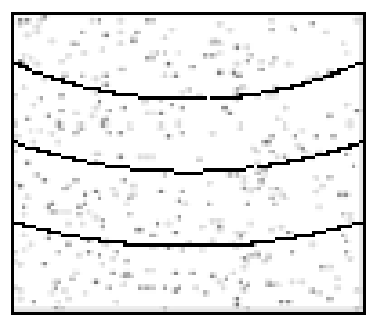

Election of candidate nodes

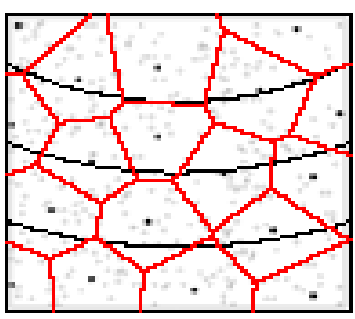

(b) Formation of clusters

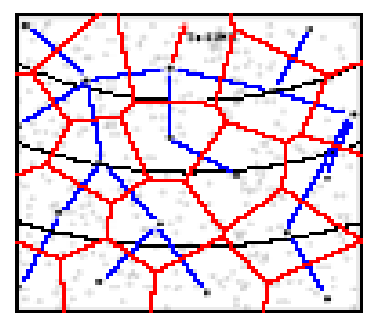

(c) Data

transmission to sink

Figure 1: Example of operation by our scheme

The above figure 1 shows operation an example of our scheme. In Figure 1(a), candidate nodes (grayed dot) are elected using threshold function by themselves. There are numerous candidate nodes more than cluster heads in Figure 1(b). Finally, Figure 1(c) shows an example of CH-to$\mathrm{CH}$ routing path to the sink for data transmission. Each cluster head transmits aggregated data to the sink along this routing path.

\section{CONCLUSION}

This paper is concerned with hierarchical routing in wireless sensor networks. The main feature of this routing technique, compared to the existing techniques, is that the election of cluster head, cluster nodes and monitoring of residual energy, etc are purely done by the cluster head. Since base station does not involve in these process, residual energy transmission over long distances is avoided, thereby reducing power consumption to much extent. It is noted that the first node death and the last node death are delayed and the overall network lifetime is prolonged

\section{REFERENCES}

[1] K.Sohraby, D. Minoli and T. Znati, Wireless Sensor Networks: Technology, Protocols, and Applications, Wiley Interscience, April 2007, pp. 1-31.

[2] F. Zhao and L. Guibas, Wireless Sensor Networks, Elsevier, 2004, pp.1-20.

[3] I. Akyildiz, W. Su, Y. Sankarasubramaniam, and E. Cayirci, "A survey on sensor networks,” IEEE Communications Magazine, Vol.. 40, No 8, pp.102-114, August 2002.

[4] C.F. García-Hernández, P. H. Ibargüengoytia-González, J. García-Hernández, and J. A. Pérez-Díaz, "Wireless Sensor Networks and Applications: a Survey," IJCSNS International Journal of Computer Science and Network Security, Vol. 7, No.3, pp. 264273, March 2007.

[5] T. Arampatzis, J. Lygeros, and S. Manesis, "A survey of applications of wireless sensors and wireless sensor networks, " in Mediterranean Conference on Control and Automation MED05, Nicosia, Cyprus, June 27-29, 2005.

[6] K. Akkaya and M. Younis, "A Survey of Routing Protocols in Wireless Sensor Networks, ”Elsevier Ad Hoc Network Journal, Vol. 3, No. 3 pp. 325-349, 2005.

[7] J.N Al-Karaki, and A.E. Kamal, "Routing techniques in wireless sensor networks: a survey,” IEEE Wireless Communications, Vol. 11, No. 6, pp.6-28, December 2004.

[8] N. Pantazis, and D. D. Vergados, "A Survey on Power Control Issues in Wireless Sensor Networks," IEEE Communications Surveys, 4th Quarter, vol. 9, No. 4, pp. 86-107, 2007. 
[9] Nikolaos A. Pantazis, Dimitrios J. Vergados, Dimitrios D. Vergados, "Increasing Intelligent Wireless Sensor Networks Survivability by Applying Energy-Efficient Schemes," in Proceedings of the 3rd IFIP Conference on Artificial Intelligence Innovations \& Applications (AIAI 2006), Athens, Greece, June 2006.

[10] W. Heinzelman, A. Chandrakasan and H. Balakrishnan, "Energy- Efficient Communication Protocol for Wireless Mi-crosensor Networks," in Proceedings of the $33 r d$ Hawaii International Conference on System Sciences (HICSS '00), January 2000.

[11] A. Manjeshwar and D. P. Agarwal, "TEEN: a routing protocol for enhanced efficiency in wireless sensor networks, " in 1st International Workshop on Parallel and. Distributed Computing Issues in Wireless Networks and Mobile Computing, San Francisco, USA, April 2001.

[12] Boselin Prabhu, SR \& Sophia, S, 2013, 'A review of energy efficient clustering algorithm for connecting wireless sensor network fields', International Journal of Engineering Research and Technology, vol. 2, no. 4, pp. 477-481.

[13] Boselin Prabhu, SR \& Sophia, S, 2013, 'Variable power energy efficient clustering for wireless sensor networks', Australian Journal of Basic and Applied Sciences, vol. 7, no. 7, pp. 423-434.

[14] Boselin Prabhu, SR \& Sophia, S, 2013, 'Capacity based clustering model for dense wireless sensor networks', International Journal of Computer Science and Business Informatics, vol. 5, no. 1, pp. 1-10.

[15] Boselin Prabhu, SR \& Sophia, S, 2013, 'Hierarchical distributed clustering algorithm for energy efficient wireless sensor networks', International Journal of Research in Information Technology, vol. 1, no. 12, pp. 45-55.

[16] Boselin Prabhu, SR \& Sophia, S, 2013, 'Real-world applications of distributed clustering mechanism in dense wireless sensor networks', International Journal of Computing Communications and Networking, vol. 2, no. 4, pp. 99-105.

[17] Boselin Prabhu, SR \& Sophia, S, 2013, 'An integrated distributed clustering algorithm for dense WSNs', International Journal of Computer Science and Business Informatics, vol. 8, no. 1, pp. 1-12.

[18] A. Manjeshwar, D.P. Agrawal, "APTEEN: a hybrid protocol for efficient routing and comprehensive information retrieval in wireless sensor networks", in Proceedings of the 2nd International Workshop on Parallel and Distributed Computing Issues in Wireless Networks and Mobile computing, Ft. Lauderdale, FL, USA, April 2002.

[19] TrueTime 1.5 - Reference Manual, Department of Automatic Control, Lund University, Sweden, Jan. 2007. Available at: http://www.control.lth.se/documents/ 2007/ ohl+07tt.pdf. 